\title{
Cetoacidose Diabética Precipitada pela COVID-19
}

\section{Diabetic Ketoacidosis Precipitated by COVID-19}

Carolina Barros ${ }^{1}$, Alexandra Malheiro¹, Mariana Martins¹, João Paulo Correia ${ }^{1}$ Miguel Perneta Santos ${ }^{1}$, Pedro Balza ${ }^{1}$

\section{RESUMO}

A COVID-19 tem um espectro de apresentação alargado, podendo variar de doença assintomática a grave. Os doentes com comorbilidades, tais como, cardiovasculares e metabólicas, têm um maior risco de desenvolver doença grave ou até mesmo complicações. A COVID-19 poderá precipitar o aparecimento de complicações metabólicas, tais como a cetoacidose diabética. Registaram-se casos de doença ligeira ou até mesmo assintomática associada a estados de cetoacidose, podendo o vírus atuar como fator precipitante de agravamento metabólico mesmo na ausência de sintomas. Torna-se imperativo, deste modo, mais estudos clínicos para compreender a correlação entre a COVID-19 e cetoacidose diabética.

PALAVRAS-CHAVE: COVID-19; Cetoacidose Diabética; SARS-CoV-2

\section{ABSTRACT}

COVID-19 has a broad spectrum of presentation, ranging from asymptomatic to severe disease. Patients with comorbidities, such as cardiovascular and metabolic, have a higher risk of developing severe disease or even complications. COVID-19 may precipitate the onset of metabolic complications such as diabetic ketoacidosis. There have been reported cases of mild or even asymptomatic disease associated with states of ketoacidosis, and the virus may act as a precipitating factor for metabolic deterioration even in the absence of symptoms. Therefore, further clinical studies are imperative to understand the correlation between COVID-19 and diabetic ketoacidosis.

KEYWORDS: COVID-19; Diabetic Ketoacidosis; SARS-CoV-2 


\section{INTRODUÇÃO}

Em dezembro de 2019 foram reportados os primeiros casos de pneumonia viral provocada pelo severe acute respiratory syndrome coronavírus 2 (SARS-CoV-2) em Wuhan, China. ${ }^{1,2}$ De acordo com os registos reportados, a diabetes mellitus (DM) foi a segunda comorbilidade $(16,2 \%)$ mais frequente nos casos graves de COVID-19. ${ }^{3}$ Esta relação parece ser bidirecional, tendo em conta o número de casos reportados de infetados pelo SARS-CoV-2 com complicações metabólicas tais como a cetoacidose diabética (CAD) em indivíduos com DM pré-existente ou até mesmo em doentes sem diagnóstico prévio. ${ }^{4}$

Em termos fisiopatológicos, especula-se que as citocinas pró-inflamatórias libertadas durante a infeção possam desempenhar um papel substancial, uma vez que a interleucina-6 encontra-se elevada tanto na CAD como na COVID-19. ${ }^{5}$ Por outro lado, a enzima conversora da angiotensina 2 (ACE2) responsável pela entrada direta do vírus nas células, expressa-se nas células beta-pancreáticas, permitindo a entrada do vírus com lesão e diminuição da função. A downregulation dos ACE2 após a entrada do vírus impede, também, a secreção de insulina pelo aumento de angiotensina. ${ }^{6}$

Este artigo tem como objetivo descrever três casos de CAD associados a infeção por SARS-CoV-2.

\section{CASOS CLÍNICOS}

\section{CASO CLÍNICO 1}

Homem de 40 anos com antecedentes pessoais de vitiligo (Tabela 1). Admitido no Hospital por quadro de confusão mental e dispneia três dias após o diagnóstico molecular de infeção por SARS-CoV-2. À admissão apresentava uma acidemia metabólica grave acompanhada de hiperglicemia de $430 \mathrm{mg} / \mathrm{dL}$ com presença de cetonúria e cetonemia de $6 \mathrm{mmol} / \mathrm{L}$ (Tabela 2). A radiografia de tórax não apresentava lesões pleuroparenquimatosas agudas e não houve necessidade de oxigenoterapia durante o internamento. Analiticamente verificou-se elevação dos parâmetros de fase aguda (PFA) com leucocitose. Foi instituída terapêutica com fluidoterapia e perfusão de insulina. Do ponto de vista de infeção SARS-CoV-2 manteve-se sempre assintomático. Apresentou estabilidade clínica ao fim de dez dias de internamento, tendo alta hospitalar medicado com o esquema terapêutico realizado durante o internamento após ter sido atingida a estabilidade clínica e analítica, nomeadamente, insulina basal glargina 12 unidades $(U)$ à noite com associação a insulina prandial glulisina $4 \mathrm{U}$ ao pequeno-almoço (PA), almoço (AL) e jantar (JA).
TABELA 1. Características dos doentes.

\begin{tabular}{|c|c|c|c|}
\hline Características & Caso 1 & Caso 2 & Caso 3 \\
\hline Idade & 40 & 72 & 26 \\
\hline Sexo & Masculino & Feminino & Masculino \\
\hline Comorbilidades & Vitiligo & $\begin{array}{l}\text { DM, HTA, } \\
\text { DRC }\end{array}$ & DM \\
\hline Grau CAD & Moderado & Moderado & Ligeiro \\
\hline $\begin{array}{l}\text { Número de dias de } \\
\text { internamento }\end{array}$ & 10 & 16 & 3 \\
\hline $\begin{array}{l}\text { Utilização de cortico- } \\
\text { terapia sistémica no } \\
\text { internamento }\end{array}$ & Não & Sim & Não \\
\hline Mortalidade & Não & Não & Não \\
\hline
\end{tabular}

$\mathrm{CAD}=$ cetoacidose metabólica; $\mathrm{DM} 2=$ diabetes mellitus tipo 2; HTA=hipertensão arterial; DRC=doença renal crónica

\section{CASO CLÍNICO 2}

Mulher de 72 anos com antecedentes pessoais de DM tipo 2, não insulinotratada (com valor de hemoglobina glicada A1C (HbA1c) prévio ao internamento de 9,8\%), Hipertensão arterial e doença renal crónica estádio 3 (Tabela 1), medicada para a DM com metformina 850 mg em associação com dapaglifozina 5 mg, duas vezes ao dia. Admitida no serviço de urgência por quadro de dispneia, febre e mialgias após contacto de alto risco com indivíduo com diagnóstico de COVID-19. Analiticamente apresentava linfopenia, elevação dos PFA, agravamento da função renal e teste molecular de pesquisa de SARS-CoV-2 positivo. Apresentava, ainda, uma acidemia metabólica moderada acompanhada de hiperglicemia 431 mg/dL (Tabela 2), e radiografia de tórax sem lesões pleuroparenquimatosas agudas. Ao segundo dia de internamento, apesar da terapêutica instituída, observou-se agravamento clínico da acidemia metabólica ( $\mathrm{pH}$ de 7,13 com bicarbonato de $12 \mathrm{mmol} / \mathrm{L}$ ), mantendo hiperglicemia de $224 \mathrm{mg} / \mathrm{dL}$. Houve necessidade de admissão na Unidade de Cuidados Intensivos (UCI), ao quarto dia de internamento, por agravamento respiratório com insuficiência respiratória tipo 1 com a presença de infiltrados algodonosos bilaterais na radiografia de tórax, resultado da evolução desfavorável da infeção e da fluidoterapia intensiva para correção do quadro metabólico. Iniciou-se oxigenoterapia de alto fluxo por cânula nasal, gestão da fluidoterapia e do perfil metabólico. Observou-se dificuldade na estabilidade clínica, com alta hospitalar apenas ao fim do décimo sexto dia de internamento. Dado o agravamento da sua função renal durante o internamento foi suspensa temporariamente a metformina e dapaglifozina, tendo tido alta medicada com insulina basal glargina de $14 \mathrm{U}$ à noite associada a $4 \mathrm{U}$ de insulina prandial glulisina ao PA, AL e JA. 
TABELA 2. Alterações analíticas apresentadas à admissão pelos doentes.

\begin{tabular}{|c|c|c|c|c|}
\hline Valores laboratoriais & Caso 1 & Caso 2 & Caso 3 & $\begin{array}{l}\text { Valor de } \\
\text { referência }\end{array}$ \\
\hline Leucócitos (x 10³/L) & 15,6 & 8,7 & 7,6 & $4,5-11$ \\
\hline Linfócitos (x 10³ $\mu / L)$ & 2,0 & 1,4 & 1,5 & $1,5-4,0$ \\
\hline Plaquetas $\left(\times 10^{3} \mu / L\right)$ & 295 & 88 & 277 & $150-450$ \\
\hline D-dímeros (ng/mL) & 655 & 1211 & - & $<200$ \\
\hline Ferritina (ng/mL) & 599 & 392 & - & $30-400$ \\
\hline $\mathrm{LDH}(\mathrm{U} / \mathrm{L})$ & 301 & 283 & 198 & $0-246$ \\
\hline Glicemia (mg/dL) & 467 & 431 & 309 & $74-110$ \\
\hline $\begin{array}{l}\text { Creatinina sérica } \\
(\mathrm{mg} / \mathrm{dL})\end{array}$ & 1,13 & 2,21 & 1,32 & $0,70-1,20$ \\
\hline Ureia (mg/dL) & 22 & 79 & 31 & $8-50$ \\
\hline $\begin{array}{l}\text { Sódio sérico ajustado } \\
(\mathrm{mEq} / \mathrm{L})\end{array}$ & 134 & 143 & 139 & $136-145$ \\
\hline $\begin{array}{l}\text { Potássio sérico } \\
(\mathrm{mEq} / \mathrm{L})\end{array}$ & 4,10 & 4,4 & 4,5 & $3,50-5,10$ \\
\hline Clorosérico (mEq/L) & 89 & 103 & 98 & $98-111$ \\
\hline PCR (mg/L) & 22 & 15 & 2 & $<6,10$ \\
\hline $\mathrm{pH}$ & 6,9 & 7,30 & 7,26 & $7,35-7,45$ \\
\hline $\mathrm{pO}_{2}(\mathrm{mmHg})$ & 102 & 98 & 100 & $80-105$ \\
\hline $\mathrm{pCO}_{2}(\mathrm{mmHg})$ & 22 & 29 & 15 & $35-45$ \\
\hline Bicarbonato , $\mathrm{mmol} / \mathrm{L})$ & 11 & 15 & 12 & $22-28$ \\
\hline $\mathrm{SaO}_{2}$ & 99 & 98 & 100 & $>95 \%$ \\
\hline Lactato (mmol/L) & 2 & 1 & 0,9 & $0,5-1$ \\
\hline $\mathrm{AG}(\mathrm{mmol} / \mathrm{L})$ & 34 & 15 & 17 & $12 \pm 4$ \\
\hline Cetonúria (mmol/L) & Positivo & Positivo & Positivo & $<0,5$ \\
\hline Cetonemia (mmol/L) & 5,4 & 4 & 6,1 & $<0,6$ \\
\hline
\end{tabular}

$\mathrm{LDH}=$ lactato desidrogenase; $\mathrm{PCR}=$ proteína $\mathrm{C}$-reativa; $\mathrm{AG}=$ anion gap

\section{CASO CLÍNICO 3}

Homem de 26 anos com antecedentes de DM tipo 1, insulino-tratado, com mau cumprimento terapêutico e consequente mau controlo metabólico (previamente ao internamento valor de HbA1C de 13\%) (Tabela 1) medicado com insulina basal glargina de $20 \cup$ à noite em associação com insulina prandial glulisina $6 \cup$ ao PA, AL e JA. Admitido no hospital ao segundo dia de infeção por SARS-CoV-2, até à data, assintomática, por quadro de vómitos alimentares. À admissão apresentava acidemia metabólica ligeira e hiperglicemia de 280 mg/dL acompanhada de cetonúria e cetonemia de 6,7 mmol/L (Tabela 2). Analiticamente verificou-se discreta elevação dos PFA com presença de linfopenia. Radiografia de tórax sem presença de lesões pleuroparenquimatosas agudas e sem necessidade de oxigenoterapia durante o internamento. Instituída terapêutica para correção da CAD com estabilidade metabólica ao fim do terceiro dia de internamento, tendo alta hospitalar assintomático com indicação para cumprimento terapêutico com incremento da insulina basal para $24 \mathrm{U}$ à noite e insulina prandial de $8 \cup$.

\section{DISCUSSÃO}

Os casos reportados demonstram que a COVID-19 poderá precipitar um quadro de CAD. Foram apresentados dois casos em indivíduos com DM já conhecida e um com diagnóstico inaugural de DM. ${ }^{6}$ A idade e outras comorbilidades apresentadas pelos doentes, assim como o valor elevado de HbA1c e a má adesão à terapêutica são fatores de risco, determinantes, para complicações metabólicas e infeciosas. ${ }^{7,8}$ A destacar, o valor de HbA1c dos últimos dois doentes (> 9\%) como possível causa de complicações infeciosas, uma vez que o ambiente hiperglicémico parece favorecer a disfunção imunológica e o aumento de virulência dos patógenos. ${ }^{9}$

A relação entre a infeção e as complicações metabólicas parece ser bidirecional, observando-se a possibilidade da infeção por SARS-CoV-2 ser um fator infecioso precipitante da CAD mesmo em doentes assintomáticos. A presença de DM complicada com CAD parece, também, conferir um risco acrescido na gravidade da COVID-19 determinando a admissão em UCl. ${ }^{10}$

A abordagem deste tipo de doentes é desafiante, tendo em conta que a ressuscitação hídrica excessiva poderá potenciar a dificuldade respiratória aguda, tornando a gestão da fluidoterapia crucial. ${ }^{4}$

São necessários mais ensaios clínicos para compreender a correlação entre a COVID-19 e a CAD.

\section{AGRADECIMENTOS/ ACKNOWLEDGMENTS}

Os autores gostariam de agradecer à restante equipa médica da Unidade e às suas coordenadoras, assim como ao serviço de Endocrinologia por todo o apoio.

\section{RESPONSABILIDADES ÉTICAS}

CONFLITOS DE INTERESSE: Os autores declaram a inexistência de conflitos de interesse na realização do presente trabalho.

FONTES DE FINANCIAMENTO: Não existiram fontes externas de financiamento para a realização deste artigo.

CONFIDENCIALIDADE DOS DADOS: Os autores declaram ter seguido os protocolos da sua instituição acerca da publicação dos dados de doentes.

CONSENTIMENTO: Consentimento do doente para publicação obtido.

PROVENIÊNCIA E REVISÃO POR PARES: Não comissionado; revisão externa por pares. 


\section{ETHICAL DISCLOSURES}

CONFLICTS OF INTEREST: The authors have no conflicts of interest to declare.

FINANCING SUPPORT: This work has not received any contribution, grant or scholarship.

CONFIDENTIALITY OF DATA: The authors declare that they have followed the protocols of their work center on the publication of data from patients.

PATIENT CONSENT: Consent for publication was obtained.

PROVENANCE AND PEER REVIEW: Not commissioned; externally peer reviewed.

\section{REFERÊNCIAS}

1. Wang D, Hu B, Hu C, Zhu F, Liu X, Zhang J, et al. Clinical Characteristics of 138 Hospitalized Patients With 2019 Novel Coronavirus-Infected Pneumonia in Wuhan, China. JAMA. 2020;323:1061-9. doi: 10.1001/jama.2020.1585. Erratum in: JAMA. 2021;325:1113.

2. Huang C, Wang Y, Li X, Ren L, Zhao J, Hu Y, et al. Clinical features of patients infected with 2019 novel coronavirus in Wuhan, China. Lancet. 2020;395:497-506. doi: 10.1016/ S0140-6736(20)30183-5.

3. Guo W, Li M, Dong Y, Zhou H, Zhang Z, Tian C, et al. Diabetes is a risk factor for the progression and prognosis of $\mathrm{CO}$ VID-19. Diabetes Metab Res Rev. 2020:e3319. doi: 10.1002/ dmrr.3319.

4. Reddy PK, Kuchay MS, Mehta Y, Mishra SK. Diabetic ketoacidosis precipitated by COVID-19: A report of two cases and review of literature. Diabetes Metab Syndr. 2020;14:1459-62. doi: 10.1016/j.dsx.2020.07.050.

5. Vaduganathan M, Vardeny O, Michel T, McMurray JJV, Pfeffer MA, Solomon SD. Renin-Angiotensin-Aldosterone System Inhibitors in Patients with Covid-19. N Engl J Med. 2020:382:1653-9. doi: 10.1056/NEJMsr2005760.

6. Chee YJ, Ng SJH, Yeoh E. Diabetic ketoacidosis precipitated by Covid-19 in a patient with newly diagnosed diabetes mellitus. Diabetes Res Clin Pract. 2020;164:108166. doi: 10.1016/j. diabres.2020.108166.

7. Zhou F, Yu T, Du R, Fan G, Liu Y, Liu Z, et al. Clinical course and risk factors for mortality of adult inpatients with $\mathrm{CO}$ VID-19 in Wuhan, China: a retrospective cohort study. Lancet. 2020;395:1054-62. doi: 10.1016/S0140-6736(20)30566-3. Erratum in: Lancet. 2020;395:1038.

8. Dhatariya KK, Glaser NS, Codner E, Umpierrez GE. Diabetic ketoacidosis. Nat Rev Dis Primers. 2020;6:40. doi: 10.1038/ s41572-020-0165-1.

9. Casqueiro J, Casqueiro J, Alves C. Infections in patients with diabetes mellitus: A review of pathogenesis. Indian J Endocrinol Metab. 2012;16 Suppl 1:S27-36. doi: 10.4103/22308210.94253.

10. Li J, Wang X, Chen J, Zuo X, Zhang H, Deng A. COVID-19 infection may cause ketosis and ketoacidosis. Diabetes Obes Metab. 2020;22:1935-41. doi: 10.1111/dom.14057. 